\title{
Copeptin concentrations during psychological stress: the Psyco study
}

\section{Judith Siegenthaler ${ }^{*}$, Carla Walti ${ }^{*}$, Sandrine Andrea Urwyler ${ }^{*}$, Philipp Schuetz ${ }^{1}$ and Mirjam Christ-Crain ${ }^{+}$}

Clinic of Endocrinology, Diabetology, and Metabolism, University Hospital Basel, Petersgraben 4, Basel CH-4031, Switzerland and ${ }^{1}$ Department of Internal Medicine, Kantonsspital Aarau, Tellstrasse, Aaraun $\mathrm{CH}-5001$, Switzerland *(J Siegenthaler, C Walti and S A Urwyler contributed equally to this work)

${ }^{\dagger} \mathrm{M}$ Christ-Crain is now at Division of Endocrinology, Diabetes and Clinical Nutrition, University Hospital Basel, Petersgraben 4, CH-4031 Basel, Switzerland

\author{
Correspondence \\ should be addressed \\ to M Christ-Crain \\ Email \\ mirjam.christ- \\ crain@unibas.ch
}

\section{Abstract}

Objective: The prognostic/diagnostic biomarker copeptin, an arginine vasopressin surrogate, reflects physical stress. Whether copeptin concentration increases upon psychological stress is unknown. We investigated psychological stress effects on copeptin secretion in healthy volunteers and patients with central diabetes insipidus (DI).

Design: A prospective observational study was conducted to study the relation between copeptin concentration and psychological stress.

Methods: A total of 20 healthy adults (ten female) and eight patients with central DI (four female) underwent the Trier Social Stress Test including, in order, 30-min waiting period, 10-min anticipation period, 10-min test period and 40-min recovery. Serum copeptin and cortisol concentrations and self-rated stress component feelings were determined in the pre-/postanticipation period, post-test period and twice post-recovery.

Results: In healthy volunteers, the median (25th-75th percentile) copeptin concentration peaked immediately during the post-test period at $5.1(3.2-7.0) \mathrm{pmol} / \mathrm{l}$, vs 3.7 (2.6-5.4) pmol/l at baseline. Over the measurement course, copeptin concentration significantly elevated (coefficient; $95 \% \mathrm{CI})(0.14 ; 0.06-0.23, P=0.002)$. The important predictors of increase in copeptin concentration were feelings of tension $(0.06 ; 0.04-0.08, P<0.001)$ and avoidance $(0.07 ; 0.04-0.10 ; P<0.001)$. Copeptin and cortisol levels were associated $(0.43 ; 0.13-0.72, P<0.005)$. Patients with DI had lower baseline concentrations (1.55 (1.2-3.1) pmol/l) when compared with healthy volunteers, $P=0.006$. Patients with DI showed no increase upon psychological stress (peak $2.15 \mathrm{pmol} / \mathrm{l}(1.5-2.28), P=0.79$ ). By contrast, cortisol values were similar in patients and volunteers. Conclusions: In healthy volunteers, copeptin levels significantly increased after psychological stress testing; this response was blunted in patients with DI.

\section{Introduction}

Arginine vasopressin (AVP) is the main hormone regulating water homoeostasis $(1,2,3)$. AVP is also a stress hormone, acting as a potent synergistic factor with corticotrophinreleasing hormone to stimulate the secretion of adrenocorticotrophic hormone and cortisol (4). Owing to preanalytical difficulties, circulating AVP is cumbersome to measure $(1,3,5)$. However, AVP is cleaved off a precursor molecule and co-secreted stoichiometrically, with three (c) 2014 European Society of Endocrinology Printed in Great Britain other peptides, among them copeptin, which is easier to measure, stable ex vivo and rapidly quantifiable by sandwich immunoassay $(1,4)$. Mirroring AVP's role in water homoeostasis, copeptin has been shown to aid in differentially diagnosing polyuria-polydipsia syndrome $(6,7)$ and hyponatraemia (8); mirroring AVP's role as a stress hormone, copeptin is a useful prognostic biomarker in several acute diseases, e.g. stroke or myocardial infarction $(9,10)$. 
Copeptin concentrations are unassociated with age, lack consistent circadian rhythm (11), increase after exercise and show slightly but significantly higher median values in men vs women (1). However, little else has been published about physiological copeptin fluctuations, despite the everincreasing literature regarding this biomarker's levels in different diseases. Thus, it is not understood as to what extent psychological stress alters copeptin secretion, knowledge that could affect this analyte's interpretation as a diagnostic or prognostic marker in clinical practice.

We hypothesised that copeptin levels would increase upon psychological stress in healthy volunteers, but not in patients with central diabetes insipidus (DI), a fluid balance disorder characterised by deficient AVP/copeptin secretion upon osmotic stimulation (12). We sought to test these hypotheses by conducting the present prospective observational study assessing copeptin kinetics in healthy volunteers and patients with DI following the Trier Social Stress Test (TSST), the most frequently used validated, standardised psychological test for stress hormone reactivity studies $(13,14)$.

\section{Subjects and methods}

\section{Subjects and ethics}

A total of 20 healthy volunteers and eight patients with central DI (four complete central DI and four partial central DI) confirmed by an adapted water deprivation test $(15,16)$ were included; their characteristics are summarised in Table 1. As per the exclusion criteria, the volunteers were in good physical health, without evidence of acute disease, history of chronic illness, BMI $>30 \mathrm{~kg} / \mathrm{m}^{2}$ or serum glucose $>7 \mathrm{mmol} / \mathrm{l}$ or serum sodium $<135$ or $>145 \mathrm{mmol} / \mathrm{l}$ at baseline. No volunteer had any psychiatric disorder. All patients with DI were on desmopressin treatment. Of the eight patients with DI, four patients had a replacement therapy with L-thyroxine, three were substituted with hydrocortisone because of hypocortisolism, two patients were on i.m. testosterone every 12 weeks for secondary hypogonadism and one patient on $\mathrm{GH}$ substitution. Apart from hormone replacement therapy, one patient was treated for hypertension and anticoagulated for deep venous thrombosis and one patient took daily tardyferon ( $n=1$ each). No other reported physical or psychiatric comorbidities.

The study was performed under the Helsinki Declaration principles, approved by the responsible Ethics Committee (Ethikkommission beider Basel, EKBB) and registered at clinicaltrials.gov (identifier NCT01866137).
Table 1 Baseline subject characteristics. Categorical variables are summarised with percentages (counts) and continuous variables with (IQR).

\begin{tabular}{|c|c|c|}
\hline & $\begin{array}{l}\text { Healthy volun- } \\
\text { teers }(n=20)\end{array}$ & $\begin{array}{c}\text { Patients with DI } \\
(n=8)\end{array}$ \\
\hline Age (years) & $22.5(20.8-25.8)$ & $46.1(38.8-47.4)$ \\
\hline Male gender & $50.0 \%(10)$ & $50.0 \%(4)$ \\
\hline Caucasian ethnicity & $100 \%(21)$ & $100 \%(8)$ \\
\hline $\begin{array}{l}\text { Alcohol consumption } \\
\text { (glasses/week) }\end{array}$ & $3.00(1.25-5.00)$ & $0.50(0.00-2.00)$ \\
\hline Current smoker & $20.0 \%(4)$ & $33.3 \%(1)$ \\
\hline Chronic diseases & $0.0 \%(0)$ & $25.0 \%(2)$ \\
\hline Psychiatric disorder & $0.0 \%(0)$ & $0.0 \%(0)$ \\
\hline Insomnia & $5 \%(1)$ & $0 \%(0)$ \\
\hline Body temperature $\left({ }^{\circ} \mathrm{C}\right)$ & $37.0(36.6-37.4)$ & $37.0(36.9-37.2)$ \\
\hline $\begin{array}{l}\text { Systolic blood pressure } \\
(\mathrm{mmHg})\end{array}$ & $114(106-125)$ & $125(110-128)$ \\
\hline $\begin{array}{l}\text { Diastolic blood pressure } \\
\quad(\mathrm{mmHg})\end{array}$ & $74.0(68.0-80.5)$ & $83.0(74.0-86.0)$ \\
\hline Heart rate $(\mathrm{bpm})$ & $65.0(56.5-74.5)$ & $67.5(60.5-73.0)$ \\
\hline Weight (kg) & $70.0(64.2-78.2)$ & $73.0(71.0-73.5)$ \\
\hline Height (m) & $1.71(1.68-1.78)$ & $1.75(1.69-1.8)$ \\
\hline BMI $\left(\mathrm{kg} / \mathrm{m}^{2}\right)$ & $22.8(21.6-25.1)$ & $21.8(21.4-22.0)$ \\
\hline Copeptin (pmol/l) & $3.70(2.55-5.40)$ & $1.55(1.2-3.1)$ \\
\hline Cortisol (nmol/l) & $431(336-517)$ & 307 (224-343) \\
\hline \multicolumn{3}{|c|}{ VAS scores for stress components } \\
\hline Discomfort & $0.0(0.0-1.0)$ & $0.0(0.0-0.5)$ \\
\hline Anxiety & $0.0(0.0-0.0)$ & $0.0(0.0-0.5)$ \\
\hline Tension & $1.0(0.0-2.0)$ & $0.0(0.0-1.5)$ \\
\hline Avoidance & $0.0(0.0-1.0)$ & $0.0(0.0-1.5)$ \\
\hline
\end{tabular}

DI, diabetes insipidus; IQR, interquartile range: 25th-75th percentile; VAS, visual analogue scale ( 0 , none at all, to 10 , very pronounced).

After full explanation of the nature and purpose of all study procedures and elements, participants gave written informed consent.

\section{Trier Social Stress Test}

The TSST protocol included a waiting period (30 min), an anticipation period (10 $\mathrm{min})$, a test period $(10 \mathrm{~min})$ and a recovery period (40 min) $(14,17)$. Right before the anticipation period, subjects were instructed regarding the tasks to be performed during the test period: delivering an ex tempore speech (first 5 min of test period) and verbally responding to a challenging arithmetic problem (second 5 min of test period), in both cases facing an audience. Subjects were then informed that the speech would be audio-recorded and video-recorded, with the recordings to, respectively, undergo voice frequency and nonverbal behaviour analyses. The test's audience comprised two trained actors, who were not to respond emotionally, but to maintain neutral facial expressions. These circumstances, as well as anticipation during speech preparation, have been shown to consistently evoke subjective stress and activate the hypothalamic-pituitary-adrenal axis and sympathoadrenomedullary system $(18,19)$. 


\section{Biochemistry}

On the testing day, volunteers and patients arrived at $0800 \mathrm{~h}$ after 24 -h abstinence from alcohol, a 10-h overnight fast and with last fluid intake allowed at $0600 \mathrm{~h}$. Patients were asked to take their last desmopressin dose before $0600 \mathrm{~h}$.

Before the 30 min waiting period, patients underwent physical examination and completed standardised anamnestic and clinical questionnaires. An i.v. catheter for blood sampling (EDTA KE/7.5, Serum Gel Z/7.5) was inserted just before the waiting period. Blood samples were collected immediately before the start of the anticipation period (baseline), after that period (+10 min), immediately after test performance $(+20 \mathrm{~min})$ and following recovery ( $+40 \mathrm{~min},+60 \mathrm{~min})$. Serum copeptin and serum cortisol concentrations were measured in all samples and to exclude osmotic stimulus, sodium, urea and glucose levels were determined at baseline and $+60 \mathrm{~min}$. Blood pressure and heart rate were measured and subjects completed a standardised stress perception questionnaire at every timepoint. In the questionnaire, feelings of discomfort, anxiety, tension and avoidance (desire to leave the situation) were each separately rated on a visual analogue scale (VAS) ranging from 0 (none) to 10 (very pronounced).
Copeptin and cortisol concentrations were measured in single batches thawed from frozen $\left(-20^{\circ} \mathrm{C}\right)$ supernatants of centrifuged serum gel tubes. Copeptin was quantitated by an immunofluorescent assay (BRAHMS CT-proAVP KRYPTOR, Thermo Scientific Biomarkers, Hennigsdorf, Germany) with a $0.9 \mathrm{pmol} / \mathrm{l}$ lower detection limit, an $<2.0 \mathrm{pmol}$ functional assay sensitivity and $<3$ to $<15 \%$ ( $<5$ to $<17 \%$ ) intra-assay (inter-assay) coefficient of variation values, depending on analyte concentrations. According to the manufacturer, the healthy adult reference range (2.5th-97.5th percentiles) was $1.2-16.4 \mathrm{pmol} / \mathrm{l}$; the median (interquartile range (IQR, 25th-75th percentiles)) value was 1.18 (1.0-1.3) pmol/l in nine patients with complete central DI (6).

Cortisol was measured by a chemiluminescence immunoassay (Siemens, Erlangen, Germany) with a reference range for morning cortisol levels of $171-536 \mathrm{nmol} / \mathrm{l}$.

Sodium, urea and glucose were quantified immediately after blood withdrawal; determinations were carried out by automated chemical analyses in the University Hospital Basel Central Laboratory.

\section{Statistical analysis}

For continuous variables, the median (IQR) is given. For categorical variables, frequencies (counts), or vice versa,

Table 2 Median and interquartile range (IQR) for copeptin, cortisol, the four stress compounds, heart rate and blood pressure in healthy volunteers $(A)$ and eight patients with DI (B).

\begin{tabular}{l} 
Variable \\
\hline (A) Healthy volunteers \\
Copeptin \\
Cortisol \\
Discomfort \\
Anxiety \\
Tension \\
Avoidance \\
Heart rate \\
Systolic blood \\
pressure (mmHg) \\
Diastolic blood \\
pressure $(\mathrm{mmHg})$ \\
(B) Patients with $\mathrm{DI}$ \\
Copeptin \\
Cortisol \\
Discomfort \\
Anxiety \\
Tension \\
Avoidance \\
Heart rate \\
Systolic blood \\
pressure (mmHg) \\
Diastolic blood \\
pressure (mmHg) \\
\end{tabular}

\begin{tabular}{c} 
Baseline $^{\mathrm{a}}$ \\
\hline \\
$3.70(2.55-5.40)$ \\
$431.0(336.0-517.0)$ \\
$0.0(0.0-1.5)$ \\
$0.0(0.0-0.0)$ \\
$0.0(0.0-1.0)$ \\
$0.0(0.0-0.0)$ \\
$57(53-64)$ \\
$109(104-127)$ \\
$72(68-78)$ \\
$1.55(1.2-3.1)$ \\
$307.0(224.0-343.0)$ \\
$0.0(0.0-0.5)$ \\
$0.0(0.0-0.5)$ \\
$0.0(0.0-1.5)$ \\
$0.0(0.0-1.5)$ \\
$62(54-70)$ \\
$116(111-121)$ \\
$75(65-78)$ \\
\end{tabular}

\begin{tabular}{c}
\hline$+20 \mathbf{~ m i n}^{\mathrm{c}}$ \\
\hline $5.10(3.20-7.00)$ \\
$521.0(484.5-696.5)$ \\
$1.0(0.0-2.0)$ \\
$0.0(0.0-1.0)$ \\
$2.0(1.0-3.5)$ \\
$2.0(0.0-2.5)$ \\
$66(62-75)$ \\
$131(118-144)$ \\
$83(76-87)$ \\
\\
$1.90(1.60-2.65)$ \\
$454.0(452.0-519.0)$ \\
$1.0(0.0-3.0)$ \\
$0.0(0.0-0.5)$ \\
$2.0(2.0-3.5)$ \\
$1.0(0.0-3.5)$ \\
$70(58-81)$ \\
$124(123-132)$ \\
$84(80-85)$ \\
\end{tabular}

\begin{tabular}{c}
\hline+60 min $^{\mathrm{d}}$ \\
\hline $3.80(2.42-5.00)$ \\
$421.5(353.8-533.2)$ \\
$0.0(0.0-1.0)$ \\
$0.0(0.0-0.0)$ \\
$0.0(0.0-0.0)$ \\
$0.0(0.0-0.0)$ \\
$60(54-68)$ \\
$113(105-125)$ \\
$74.5(66-78)$ \\
\\
$2.15(1.5-2.28)$ \\
$311.5(299.0-325.5)$ \\
$0.0(0.0-0.0)$ \\
$0.0(0.0-0.0)$ \\
$0.0(0.0-0.0)$ \\
$0.0(0.0-0.0)$ \\
$65(57-74)$ \\
$116(107-124)$ \\
$74(68-76)$ \\
\end{tabular}

${ }^{\text {a }}$ Pre-anticipation period.

bost-anticipation period.

cPost-test period.

dPost-recovery.

\begin{tabular}{c}
\hline $\mathbf{4 0} \mathbf{~ m i n}^{\mathrm{d}}$ \\
\hline $3.70(2.45-5.30)$ \\
$481.0(428.5-627.0)$ \\
$0.0(0.0-1.0)$ \\
$0.0(0.0-0.0)$ \\
$0.0(0.0-0.5)$ \\
$0.0(0.0-0.0)$ \\
$59(56-67)$ \\
$114(104-123)$ \\
$76.50(70-79)$ \\
\\
$2.10(1.35-2.5)$ \\
$420.0(396.0-567.0)$ \\
$0.0(0.0-1.5)$ \\
$0.0(0.0-0.5)$ \\
$0.0(0.0-0.5)$ \\
$0.0(0.0-0.0)$ \\
$60(54-72)$ \\
$118(109-123)$ \\
$79(74-80)$ \\
\end{tabular}

\begin{tabular}{c}
+10 min $^{\mathrm{b}}$ \\
\hline $4.00(2.80-5.70)$ \\
$472.0(414.0-555.0)$ \\
$2.0(0.5-3.5)$ \\
$1.0(0.0-2.0)$ \\
$3.0(2.0-4.0)$ \\
$2.0(1.0-3.5)$ \\
$66(61-77)$ \\
$120(113-136)$ \\
$75(69-83)$ \\
\\
$1.90(1.38-2.55)$ \\
$426.0(359.0-459.0)$ \\
$1.5(0.0-3.0)$ \\
$1.0(0.0-3.5)$ \\
$2.0(1.0-5.5)$ \\
$1.5(1.0-4.0)$ \\
$65(62-80)$ \\
$122(114-140)$ \\
$80(77-83)$ \\
\end{tabular}




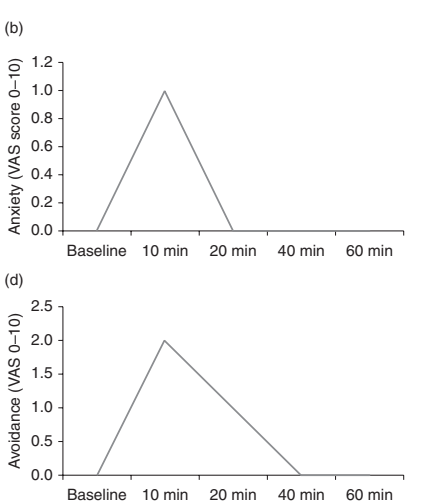

\section{Figure 1}

Subjective stress perception: VAS scores for (a) discomfort, (b) anxiety, (c) tension and (d) avoidance at different timepoints before, during and after the TSST. TSST, Trier Social Stress Test; VAS, visual analogue scale (0, none, to 10 , very pronounced.).

are reported. To compare serial copeptin or serial cortisol concentrations, or both, mixed models were used to account for non-independence of measurements within subjects; hormone levels were log-transformed before inclusion. The categorical variable treatment (TSST) was used as a fixed effect. For every subject, separate intercepts and separate slopes were fitted for each measurement time. The models used a within-group (serial) autocorrelation structure, leading to considerably better model fits than did ignoring within-group correlations. For each model and fixed effect, the coefficient, the approximate 95\% CI, the denominator degrees of freedom, the $t$-statistic and the associated $P$ value were calculated. Testing was two tailed; $P \leq 0.05$ was deemed significant.

\section{Results}

As reflected by median VAS scores for feelings of discomfort, anxiety, tension and avoidance, subjective stress perception peaked at the end of the anticipation period (+10 min measurement point) (Table 2 and Fig. 1a, b, c and d). Median copeptin and cortisol concentrations peaked at the end of the test period ( +20 min timepoint) (Table 2 and Fig. $2 \mathrm{a}$ and $\mathrm{b}$ ), respectively, rising by $1.4 \mathrm{pmol} / \mathrm{l}(38 \%)$ and $90 \mathrm{nmol} / 1$ (21\%) from baseline.

Over the measurement course, the TSST significantly increased copeptin levels (coefficient; 95\% CI) (0.14; 0.06-0.23, $P=0.002$ ) (Table 3). The most important predictors of increase in copeptin concentration were feelings of tension (0.06; 0.04-0.08, $P<0.001)$ and avoidance $(0.07 ; 0.04-0.10, P<0.001)$. The TSST also significantly raised cortisol values $(0.16$; $0.09-0.23, P<0.001)$, which result was mainly influenced by feelings of tension (0.03; 0.01-0.05, $P=0.006)$. Levels of the two hormones were significantly associated (0.43; 0.13-0.72, $P<0.005)$. Glucose, urea and sodium levels remained unchanged during the measurement course (data not shown).

Patients with DI in comparison with healthy volunteers had lower baseline copeptin levels $(1.55 ; 1.2-3.1$ vs $3.7 ; 2.9-$ $5.4, P=0.006$ ) (Table 1 ) and showed no significant increase (peak 2.15 (1.5-2.28) pmol/l) upon psychological stress, $P=0.79$ (Table 2 and Fig. 2a). By contrast, cortisol baseline levels and kinetics upon psychological stress were comparable to those in healthy volunteers (Table 2 and Fig. 2b). Owing to hydrocortisone treatment in three patients with DI, only five patients were considered for analysis of cortisol levels. Subjective stress perception was also similar compared with that of healthy volunteers (data not shown).

\section{Discussion}

This study's main novel finding was that copeptin levels, in parallel to cortisol levels, increased upon psychological stress in healthy volunteers, especially as embodied by feelings of tension and avoidance (desire for flight). This stands in contrast to patients with DI, where only an increase in cortisol concentration but no change in copeptin concentration was observed upon psychological stress.

This finding would appear to have two clinical implications. First, copeptin values may need to be interpreted cautiously in psychologically stressed patients. This caveat may be especially important in the emergency setting, where copeptin levels are, for example, applied for rapid rule-out of acute myocardial infarction (10).

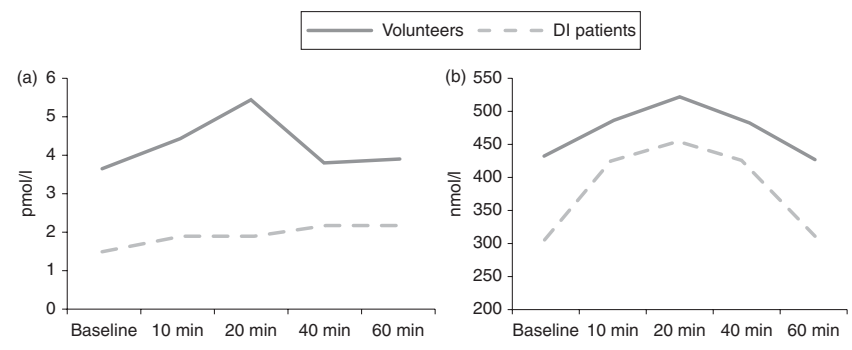

\section{Figure 2}

Temporal progression of (a) copeptin concentrations and (b) cortisol concentration in 20 healthy adult volunteers and eight adults with central DI. Measurement time 0 (baseline) was before the TSST anticipation period, time $+10 \mathrm{~min}$ was at the end of that period, time +20 min was at the end of the test period and times +40 and $+60 \mathrm{~min}$ were after the recovery period. DI, diabetes insipidus. 
Table 3 Effect of stress test on copeptin and cortisol levels in healthy volunteers.

\begin{tabular}{|c|c|c|}
\hline $\begin{array}{l}\text { Exposure } \\
\text { variables }\end{array}$ & $\begin{array}{l}\text { Effect of stress test on } \\
\text { log-transformed marker } \\
\text { level (coefficient, } 95 \% \mathrm{Cl} \text { ) }\end{array}$ & $\boldsymbol{P}$ \\
\hline \multicolumn{3}{|l|}{ Copeptin } \\
\hline Overall stress & $0.14 ;-0.06$ to 0.23 & 0.002 \\
\hline Discomfort & $0.04 ;-0.00$ to 0.07 & 0.03 \\
\hline Anxiety & $0.05 ;-0.00$ to 0.11 & 0.07 \\
\hline Tension & $0.06 ; 0.04$ to 0.08 & $<0.001$ \\
\hline Avoidance & $0.07 ; 0.04$ to 0.10 & $<0.001$ \\
\hline \multicolumn{3}{|l|}{ Cortisol } \\
\hline Overall stress test & $0.16 ; 0.09$ to 0.23 & $<0.001$ \\
\hline Discomfort & $0.02 ;-0.01$ to 0.04 & 0.19 \\
\hline Anxiety & $0.03 ;-0.01$ to 0.08 & 0.15 \\
\hline Tension & $0.03 ; 0.01$ to 0.05 & 0.006 \\
\hline Avoidance & $0.03 ;-0.00$ to 0.06 & 0.04 \\
\hline
\end{tabular}

Copeptin cut-off values have also been suggested for diagnosis and treatment guidance in the polyuriapolydipsia syndrome (6). Altered copeptin levels upon psychological stress call into question fixed cut-offs in clinical routine, where blood is often taken while patients experience anxiety and tension.

Secondly, copeptin might represent a sensitive alternative to adrenocorticotrophic hormone or cortisol to measure individual perception of psychological stress. In contrast to cortisol, no cross-reactivity with other steroid hormones is known for copeptin. While cortisol levels follow a circadian rhythm, no consistent circadian rhythm was found for copeptin levels thus far, although tested only in a small sample of patients $(1,11)$. Moreover, copeptin was shown to mirror individual stress levels more subtle than cortisol (20).

Recently, the role of vasopressin in social behaviour and stress has been investigated and shows that social information is partly processed by vasopressin $(21,22)$. It has been shown that the genetic mechanisms of interindividual variation in the vasopressin-oxytocin pathway influence human social interaction (21). AVP therefore emerged together with oxytocin as a target for novel treatment approaches for mental disorders characterised by social dysfunction. In two studies, the intranasal administration of AVP resulted in a significant increase in salivary cortisol and heart rate compared with placebo in the TSST (23). This finding suggests that AVP is a key player in the sensation and regulation of stress and social threat; therefore, AVP antagonists have been considered for the treatment of major depression and may also be helpful in the treatment of stress-related disorders and disorders that are characterised by interpersonal violence, such as antisocial personality disorders (21).
Patients with central DI have endogenous AVP/ copeptin deficiency upon osmotic stimulation. Even in an absence of change in osmolality, physical stress normally results in a rise in AVP secretion secondary to increased hypothalamic-pituitary-adrenal axis activity (24). In this study, we showed that patients with central DI could not secrete copeptin in situations of psychological stress. Interestingly, however, their subjective stress perception and cortisol secretion seemed to be normal. This finding of normal cortisol response to acute stress in adults with central DI agrees with a recent work in children with this condition (25).

Our study had limitations. First, it had a small, singlecentre sample. However, as baseline fasting copeptin levels in our volunteer cohort were comparable to those observed in other healthy volunteers $(1,3)$, we consider it probable that our results in healthy volunteers were robust. The sample size of our patients with DI $(n=8)$ is rather small and needs validation in further studies; however, our results are of interest showing for the first time that patients with central DI and AVP/copeptin deficiency are not able to react to psychological stress with an increase in AVP/copeptin concentration but show a normal cortisol response. However, as a further limitation, we cannot fully exclude an influence of exogenous desmopressin administration on copeptin levels in patients with DI. Secondly, the volunteer sample predominantly comprised young adults, whereas in clinical practice, copeptin is measured in all age groups. However, copeptin concentrations have been shown not to be associated with age $(1,26)$; we therefore consider different results in an older population to be unlikely.

Thirdly, we relied on within-subject comparison. We decided against a control group not undergoing psychological stress testing, as previous data indicated no circadian rhythm of copeptin (1).

In conclusion, in healthy volunteers, median copeptin levels slightly but significantly rose upon psychological stress, stimulated mainly by feelings of tension and avoidance. This finding suggests that it may be important to consider patients' psychological stress when interpreting copeptin values. In contrast to healthy volunteers, patients with central DI appear to be unable to secrete copeptin upon psychological stress, but retain normal cortisol response.

\section{Declaration of interest}

$M$ Christ-Crain and $P$ Schuetz have received honoraria from Thermo Scientific Biomarkers (formerly BRAHMS AG), the copeptin assay developer/manufacturer, to fulfil speaking engagements and travel support to attend medical meetings. Thermo has also provided research grants to the Division of Endocrinology, Diabetes and Clinical Nutrition, University 
Hospital Basel and the Department of Internal Medicine, Kantonsspital Aarau. The authors have no other declarations of interest.

\section{Funding}

This research did not receive any specific grant from any funding agency in the public, commercial or not-for-profit sector.

\section{Acknowledgements}

The authors thank Robert Marlowe, Spencer-Fontayne Corporation, Jersey City, NJ, USA, for editing this manuscript.

\section{References}

1 Morgenthaler NG, Struck J, Alonso C \& Bergmann A. Assay for the measurement of copeptin, a stable peptide derived from the precursor of vasopressin. Clinical Chemistry 200652 112-119. (doi:10.1373/ clinchem.2005.060038)

2 Balanescu S, Kopp P, Gaskill MB, Morgenthaler NG, Schindler C \& Rutishauser J. Correlation of plasma copeptin and vasopressin concentrations in hypo-, iso-, and hyperosmolar States. Journal of Clinical Endocrinology and Metabolism 201196 1046-1052. (doi:10.1210/ jc.2010-2499)

3 Szinnai G, Morgenthaler NG, Berneis K, Struck J, Muller B, Keller U \& Christ-Crain M. Changes in plasma copeptin, the c-terminal portion of arginine vasopressin during water deprivation and excess in healthy subjects. Journal of Clinical Endocrinology and Metabolism 200792 3973-3978. (doi:10.1210/jc.2007-0232)

4 Katan M \& Christ-Crain M. The stress hormone copeptin: a new prognostic biomarker in acute illness. Swiss Medical Weekly 2010140 w13101. (doi:10.4414/smw.2010.13101)

5 Robertson GL, Mahr EA, Athar S \& Sinha T. Development and clinical application of a new method for the radioimmunoassay of arginine vasopressin in human plasma. Journal of Clinical Investigation $1973 \mathbf{5 2}$ 2340-2352. (doi:10.1172/JCI107423)

6 Fenske W, Quinkler M, Lorenz D, Zopf K, Haagen U, Papassotiriou J, Pfeiffer AF, Fassnacht M, Stork S \& Allolio B. Copeptin in the differential diagnosis of the polydipsia-polyuria syndrome - revisiting the direct and indirect water deprivation tests. Journal of Clinical Endocrinology and Metabolism 201196 1506-1515. (doi:10.1210/jc.2010-2345)

7 Katan M, Morgenthaler NG, Dixit KC, Rutishauser J, Brabant GE, Muller B \& Christ-Crain M. Anterior and posterior pituitary function testing with simultaneous insulin tolerance test and a novel copeptin assay. Journal of Clinical Endocrinology and Metabolism 200792 2640-2643. (doi:10.1210/jc.2006-2046)

8 Fenske W, Stork S, Blechschmidt A, Maier SG, Morgenthaler NG \& Allolio B. Copeptin in the differential diagnosis of hyponatremia. Journal of Clinical Endocrinology and Metabolism 200994 123-129. (doi:10.1210/jc.2008-1426)

9 Tu WJ, Dong X, Zhao SJ, Yang DG \& Chen H. Prognostic value of plasma neuroendocrine biomarkers in patients with acute ischaemic stroke. Journal of Neuroendocrinology 201325 771-778. (doi:10.1111/jne.12052)

10 Reichlin T, Hochholzer W, Stelzig C, Laule K, Freidank H, Morgenthaler NG, Bergmann A, Potocki M, Noveanu M, Breidthardt T et al. Incremental value of copeptin for rapid rule out of acute myocardial infarction. Journal of the American College of Cardiology 2009 54 60-68. (doi:10.1016/j.jacc.2009.01.076)

11 Darzy KH, Dixit KC, Shalet SM, Morgenthaler NG \& Brabant G. Circadian secretion pattern of copeptin, the C-terminal vasopressin precursor fragment. Clinical Chemistry 201056 1190-1191. (doi:10.1373/clinchem.2009.141689)

12 Katan M, Muller B \& Christ-Crain M. Copeptin: a new and promising diagnostic and prognostic marker. Critical Care 200812117. (doi:10.1186/cc6799)

13 Dickerson SS \& Kemeny ME. Acute stressors and cortisol responses: a theoretical integration and synthesis of laboratory research. Psychological Bulletin 2004130 355-391. (doi:10.1037/0033-2909.130.3.355)

14 Birkett MA. The Trier Social Stress Test protocol for inducing psychological stress. Journal of Visualized Experiments 2011563238. (doi:10.3791/3238)

15 Zerbe RL \& Robertson GL. A comparison of plasma vasopressin measurements with a standard indirect test in the differential diagnosis of polyuria. New England Journal of Medicine 1981305 1539-1546. (doi:10.1056/NEJM198112243052601)

16 Milles JJ, Spruce B \& Baylis PH. A comparison of diagnostic methods to differentiate diabetes insipidus from primary polyuria: a review of 21 patients. Acta Endocrinologica 1983104 410-416.

17 Kirschbaum C, Pirke KM \& Hellhammer DH. The 'Trier Social Stress Test' - a tool for investigating psychobiological stress responses in a laboratory setting. Neuropsychobiology 199328 76-81. (doi:10.1159/000119004)

18 Kelly MM, Tyrka AR, Anderson GM, Price LH \& Carpenter LL. Sex differences in emotional and physiological responses to the Trier Social Stress Test. Journal of Behavior Therapy and Experimental Psychiatry 2008 39 87-98. (doi:10.1016/j.jbtep.2007.02.003)

19 Jonsson P, Wallergard M, Osterberg K, Hansen AM, Johansson G \& Karlson B. Cardiovascular and cortisol reactivity and habituation to a virtual reality version of the Trier Social Stress Test: a pilot study. Psychoneuroendocrinology 201035 1397-1403. (doi:10.1016/ j.psyneuen.2010.04.003)

20 Katan M, Morgenthaler N, Widmer I, Puder JJ, Konig C, Muller B \& Christ-Crain M. Copeptin, a stable peptide derived from the vasopressin precursor, correlates with the individual stress level. Neuro Endocrinology Letters 200829 341-346.

21 Meyer-Lindenberg A, Domes G, Kirsch P \& Heinrichs M. Oxytocin and vasopressin in the human brain: social neuropeptides for translational medicine. Nature Reviews. Neuroscience 201112 524-538. (doi:10.1038/ nrn3044)

22 Tobin VA, Hashimoto H, Wacker DW, Takayanagi Y, Langnaese K, Caquineau C, Noack J, Landgraf R, Onaka T, Leng G et al. An intrinsic vasopressin system in the olfactory bulb is involved in social recognition. Nature 2010464 413-417. (doi:10.1038/nature08826)

23 Ebstein RP, Israel S, Lerer E, Uzefovsky F, Shalev I, Gritsenko I, Riebold M, Salomon S \& Yirmiya N. Arginine vasopressin and oxytocin modulate human social behavior. Annals of the New York Academy of Sciences 2009 1167 87-102. (doi:10.1111/j.1749-6632.2009.04541.x)

24 Donald RA, Alexander SL, Ellis MJ, Evans MJ, Livesey JH \& Espiner EA. Regulation of corticotrophin secretion. Clinical Science 199588 4-7.

25 Bas F, Darendeliler F, Tatli B, Unal M \& Kayserilioglu A. Corticotropin and cortisol response to maximal exercise testing in central diabetes insipidus. Pediatrics International 200749 53-57. (doi:10.1111/ j.1442-200X.2007.02320.x)

26 Morgenthaler NG, Struck J, Jochberger S \& Dunser MW. Copeptin: clinical use of a new biomarker. Trends in Endocrinology and Metabolism 200819 43-49. (doi:10.1016/j.tem.2007.11.001)

Received 27 May 2014

Revised version received 9 September 2014

Accepted 23 September 2014 